\title{
Diseño de investigación cualitativa: análisis de los factores más relevantes para implementar la enseñanza virtual en educación secundaria
}

\author{
Dolly Britton Jiménez \\ Docente del cuerpo de profesores de enseñanza secundaria de Castilla-La Mancha (España) \\ dly.britton@gmail.com | https://orcid.org/0000-0002-9956-4513 \\ Carolina Casal Funcasta \\ Docente del cuerpo de profesores de enseñanza secundaria de la Xunta de Galicia (España) \\ casalfuncasta@edu.xunta.gal | https://orcid.org/0000-0003-0330-7166
}

Mariano Urraco Solanilla

Profesor de Sociología del Departamento de Educación de la Universidad a Distancia de Madrid, UDIMA (España)

mariano.urraco@udima.es | https://orcid.org/0000-0002-5839-0019

\section{Extracto}

El presente artículo constituye un diseño de investigación orientado a analizar los problemas surgidos en un instituto de educación secundaria durante el tercer trimestre del curso 20192020, momento en que la pandemia provocada por la COVID-19 hizo necesario que los centros educativos continuaran su actividad de manera virtual. La propuesta de investigación planteada posibilitaría la obtención de datos sobre el grado de preparación para la enseñanza a distancia de la comunidad educativa del instituto tomado como caso de estudio. Así pues, este trabajo etnográfico permitiría, una vez desarrollado, conocer en detalle la situación vivida en el instituto y ayudaría al diseño de propuestas educativas generalizables para cualquier centro educativo de rasgos similares al analizado en este caso de estudio. Los resultados de la investigación propuesta en este artículo vendrían a aportar información sobre los factores que hay que tener en cuenta para poder diseñar propuestas metodológicas de enseñanza secundaria virtual.

Palabras clave: enseñanza secundaria; competencias TIC (tecnologías de la información y la comunicación); educación alternativa; sistema educativo español. 


\title{
Qualitative research design: analysis of the most relevant factors for implementing virtual education in secondary education
}

\author{
Dolly Britton Jiménez \\ Carolina Casal Funcasta \\ Mariano Urraco Solanilla
}

\begin{abstract}
The following article, conceived as a research proposal, aims at analysing the difficulties encountered at a high school during the third term of the academic year 2019-2020, when the COVID-19 pandemic made it necessary for schools to continue their activity virtually. This research proposal would make it possible to obtain data about the degree of preparation for distance learning of the educational community chosen as case study. Once developed, this ethnographic research would allow to know the situation experienced at that high school in detail, and it would help to design generalizable educational proposals suitable for any school of similar features to those of the one selected in this case study. The results of the research suggested in this article would provide information about the factors to consider when designing methodological proposals for distance learning at secondary levels.
\end{abstract}

Keywords: secondary education; ICTs (information and communication technologies) competences; homeschooling; Spanish educational system.

Citation: Britton Jiménez, D., Casal Funcasta, C. and Urraco Solanilla, M. (2021). Qualitative research design: analysis of the most relevant factors for implementing virtual education in secondary education. Tecnología, Ciencia y Educación, 19, 147-170. https://doi.org/10.51302/tce.2021.620 


\section{Sumario}

1. Introducción

2. Marco teórico: potencialidad de la educación secundaria virtual

2.1. Virtualización de la educación: contexto general

2.2. Propuestas pedagógicas de enseñanza virtual

2.3. Papel de las familias en la educación secundaria virtual

3. Desarrollo: diseño de investigación cualitativa

3.1. Objetivos

3.2. Estudio de caso etnográfico

3.3. Diseño de investigación

3.4. Plan de explotación de los resultados

4. Conclusiones

Referencias bibliográficas

Nota: este artículo es una versión del trabajo fin de máster realizado por las estudiantes Dolly Britton Jiménez y Carolina Casal Funcasta, bajo la dirección del profesor Mariano Urraco Solanilla, en el marco del Máster en Tecnología Educativa de la UDIMA. 


\section{Introducción}

Dos son los motivos fundamentales que sustentan la importancia del presente trabajo. Por una parte, en los últimos años ha aumentado exponencialmente el aprendizaje no presencial. La rapidez con la que se está incrementando el número de instituciones de formación superior a distancia nos hace anticipar que, probablemente, sea cuestión de tiempo que la enseñanza en línea se convierta en una práctica habitual también en otros niveles como el de la educación secundaria obligatoria (ESO) o el bachillerato. Por otra parte, el cierre de todos los centros educativos del país como medida de contención de la pandemia provocada por la COVID-19 obligó al conjunto de la comunidad educativa a actuar con rapidez para continuar garantizando el derecho a la educación en España durante los últimos meses del curso 2019-2020. Esta tarea recayó en manos de los miembros profesionales de dicha comunidad educativa (administraciones educativas, equipos directivos, profesorado, personal de orientación y auxiliares) sin un procedimiento o reglamento previamente diseñado que garantizase su correcta aplicación. Paralelamente, la labor educativa también pasó a ser responsabilidad de personas sin formación específica para la docencia (familias con distintas situaciones laborales, económicas, sociales y académicas) y del propio alumnado (estudiantes, por descontado, de distintas edades, necesidades, recursos y competencias tecnológicas). Las dificultades a las que gran parte de la sociedad se enfrentó en esta ardua labor de mitigar los inconvenientes de la suspensión de las clases presenciales, así como las soluciones y estrategias que se fueron descubriendo y aplicando, suponen una información -en mayor o menor medida- novedosa en algunos ámbitos educativos, entre ellos, el de la educación primaria y secundaria online.

La reflexión de los desafíos vividos cuando una persona se inicia en la enseñanza-aprendizaje online o virtual es, como ya decían algunos de los pioneros en esta materia, necesaria para definir los distintos roles que deben desempeñar todos sus participantes (Adell y Sales, 1999). Por ello, la investigación que aquí se propone pretendería contribuir al desarrollo de la literatura, concretamente, en la de la educación secundaria (ESO y bachillerato). De esta manera, el análisis de cómo se impartió docencia virtual en el IES Impulsa ${ }^{1}$

1 Tanto el nombre como todo lo relativo al instituto objeto de estudio es ficticio. El diseño se basa en un centro real, con las características que se recogen en este documento, pero se ha optado por mantener el anonimato de dicho centro cambiando su localización y recurriendo al uso de un nombre inventado. 
y de qué modo se usaron los dispositivos tecnológicos en todos los ámbitos y niveles durante el periodo de confinamiento proporcionaría información detallada que fundamentase el diseño metodológico y la reglamentación oficial de planes educativos de una educación secundaria a distancia que ya está siendo demandada por la sociedad y por las circunstancias (Xunta de Galicia, 2020).

Como veremos en las próximas páginas, la educación secundaria virtual, en muchos aspectos, ya existe, por lo que tenemos que estar preparados para un posible escenario futuro en el que su implantación sea generalizada y las autoridades educativas tengan que regular este tipo de enseñanza en España. He ahí la importancia de llevar a cabo, en la práctica, la investigación que planteamos ahora como diseño

La COVID-19 ha desencadenado, en el último año, toda una serie de actuaciones poco planificadas por parte de las Administraciones educativas, de los equipos directivos y de los propios docentes o propuesta: es necesario conocer y analizar los factores que intervienen antes de actuar. Paradójicamente, sin embargo, la realidad ha sido otra, pues la COVID-19 ha desencadenado, en el último año, toda una serie de actuaciones poco planificadas por parte de las Administraciones educativas, de los equipos directivos y de los propios docentes. Estas decisiones, a falta de estudios de investigación específicos, constituyen los antecedentes literarios de la investigación que nos ocupa, por lo que creemos conveniente sintetizarlas muy brevemente organizándolas en tres bloques:

- Las relacionadas con los recursos tecnológicos (muchos institutos y Administraciones educativas optaron por prestar dispositivos electrónicos y routers wifi a aquellas familias que los necesitaban para poder llevar a cabo la educación obligatoria desde casa) (Nadeu, 2020; Vallespín, 2020b).

- Las referentes a la cantidad y tipología de las tareas (no hubo acuerdo respecto a si se debía llevar el mismo ritmo que en la enseñanza presencial, pero la consigna predominante fue la de flexibilizar metodologías e impartir lo «imprescindible») (Orden Educación y Formación Profesional [EFP], 2020).

- Las relacionadas con la actividad docente, con la forma de impartir clase, de motivar a los estudiantes y de evaluar los contenidos (no se establecieron unos mínimos comunes, sino que cada instituto, y, en muchos casos, cada docente, tuvo potestad para escoger su modo, plataforma y horario de dar clase) (Consejería de Educación, Cultura y Deportes de Castilla-La Mancha, 2020).

Estas decisiones, y, en general, todo el periodo de educación obligatoria virtual, pueden -y deben- ser analizados como si se tratase de un «programa piloto» o «periodo de detección de errores", extrayendo datos que sirvan para tomar decisiones futuras fundamentadas y para diseñar planes educativos y metodológicos de enseñanza secundaria a distancia basados en el proceso de «experimentación-error» llevado a cabo en el periodo de 
confinamiento de 2020. A la espera de dichos planes, esta propuesta de investigación educativa sirve de guía, por tanto, a los encargados de diseñarlos, por cuanto ofrece el diseño de un estudio de caso cuya implementación podría aportar conocimiento sobre los elementos que deberían ser tomados en consideración en la elaboración de los mencionados planes de actuación de cara al futuro.
Esta propuesta ofrece el diseño de un estudio de caso cuya implementación podría aportar conocimiento sobre los elementos que deberían ser tomados en consideración en la elaboración de planes de actuación de cara al futuro

\section{Marco teórico: potencialidad de la educación secundaria virtual}

\subsection{Virtualización de la educación: contexto general}

En la Ley orgánica 14/1970, de 4 de agosto, general de educación y financiamiento de la reforma educativa (LGE, 1970, p. 12.532) ya se recogía que, para garantizar el derecho de la educación a quienes «no puedan asistir regularmente a los centros ordinarios», el ministerio y los organismos competentes reglamentarían «las modalidades de enseñanza por correspondencia, radio y televisión y el establecimiento de cursos nocturnos en periodo no lectivo». Desde entonces, el auge de las nuevas tecnologías de la comunicación ha sustituido la radio, la televisión y el correo por la enseñanza «en línea», y, actualmente, 6 de las 83 universidades del país imparten docencia enteramente a distancia (Ministerio de Universidades, 2020) y 51 ofrecen estudios online (U-Multirank, s. f.). Por otra parte, en el ámbito no universitario, los 14.289 alumnos de formación profesional (grado medio y superior) del curso académico 2008-2009 (Instituto Nacional de Estadística [INE], 2018) pasaron a ser 96.816 en el curso 2019-2020 (Ministerio de Educación y Formación Profesional [MEFP], 2019). Estos datos hacen visible la importancia que, en las últimas décadas, ha adquirido la enseñanza a distancia en dos niveles educativos distintos y abren la puerta a nuevas incógnitas, entre ellas, si llegará al ámbito de la educación secundaria. Como respuesta, la legislación española estipula que dicha etapa educativa -al igual que la educación primariadebe impartirse de manera presencial a todo el alumnado menor de 18 años. No obstante, encontramos modelos de educación que muestran cómo la educación virtual se abre paso en estas etapas.

El ejemplo tal vez más significativo es el Centro Integrado de Enseñanzas Regladas a Distancia (CIERD), creado en 2015 y perteneciente al Centro para la Innovación y el Desarrollo de la Educación a Distancia (CIDEAD), dependiente del MEFP. En el CIERD, se imparte educación primaria, secundaria y bachillerato, de manera virtual, a aquellas personas con nacionalidad española residentes en el extranjero "que hayan cursado estudios reglados españoles», así como a estudiantes residentes en España «en circunstancias excepcionales que les impidan» asistir a un centro de su comunidad autónoma -en este caso, previa 
autorización expresa de la Administración educativa y del CIDEAD- (CIERD, s. f., párr. 6). En el curso 2016-2017, el CIERD contó con 684 estudiantes de educación secundaria (MEFP, 2018a); en el curso escolar 2017-2018, último del que se dispone de datos, esta cifra aumentó hasta los 1.140 estudiantes matriculados (MEFP, 2018b).

Para este y todos los demás centros educativos españoles, en 2018, la Agencia Española de Protección de Datos (AEPD) publicó el Informe sobre la utilización por parte de profesores y alumnos de aplicaciones que almacenan datos en la nube con sistemas ajenos a las plataformas educativas. La necesidad de tal informe evidencia la incursión de los miembros de la educación obligatoria en el mundo digital, tanto por decisión propia en el diseño de la actividad docente como por la necesidad de alcanzar uno de los 12 objetivos que la Ley orgánica 2/2006, de 3 de mayo, de educación (LOE, 2006) estipula para el sistema educativo español: «La plena inserción del alumnado en la sociedad digital y el aprendizaje de un uso seguro de los medios digitales» (p. 16). Las conclusiones de dicho informe de la AEPD inciden en los riesgos que supone el uso de determinadas aplicaciones y plataformas educativas en cuanto a la recopilación de datos personales y a la «elaboración de perfiles de aprendizaje, preferencias o comportamiento de menores de edad por parte de los responsables de las aplicaciones» (AEPD, 2018, p. 7). La solución propuesta por la AEPD, sin embargo, no pasa por prohibir el uso de tales aplicaciones, sino por evaluarlas siguiendo unas consideraciones específicas y, entre otras medidas, establecer procedimientos por los cuales los centros educativos deban solicitar autorizaciones de uso (AEPD, 2018).

Prueba de la tendencia a digitalizar algunos aspectos de la educación secundaria es la existencia de múltiples páginas web y plataformas dedicadas a compartir material educativo en abierto. Ponemos como ejemplos más representativos el proyecto Agrega 2, la red Procomún y la plataforma e-Twinning. El primero, Agrega 2, fue creado por el Ministerio de Educación, Cultura y Deporte ${ }^{2}$, por las comunidades
Prueba de la tendencia a digitalizar algunos aspectos de la educación secundaria es la existencia de múltiples páginas web y plataformas dedicadas a compartir material educativo en abierto autónomas y por la entidad Red.es (dependiente del Ministerio de Asuntos Económicos y Transformación Digital) y se trata de una plataforma implantada en todas las Administraciones educativas del país que ofrece contenidos educativos a disposición del profesorado y alumnado de la enseñanza primaria y secundaria. El segundo ejemplo, Procomún, dependiente del MEFP, también es una red de contenidos educativos en abierto que, además, constituye una plataforma de contacto entre los distintos miembros de la comunidad educativa. A su vez, Procomún enlaza con más redes de recursos en abierto, como

2 En virtud del Real Decreto 355/2018, de 6 de junio, por el que se reestructuran los departamentos ministeriales, a partir de esa fecha corresponde al Ministerio de Educación y Formación Profesional la propuesta y ejecución de la política del Gobierno en materia educativa y de formación profesional. 
Europeana, que ofrece archivos de bibliotecas y museos para la educación, el ocio y la investigación. Sobrepasando las fronteras españolas, la plataforma cofinanciada por Erasmus+, e-Twinning, demuestra la capacidad que tienen los centros educativos no universitarios de organizar, entre muchas otras cosas, proyectos educativos virtuales en colaboración con escuelas e institutos de todo el mundo.

Interesa, asimismo, mencionar la página web que el ministerio ha puesto en marcha para ayudar a los miembros de la comunidad educativa durante el periodo de confinamiento: \#AprendoEnCasa, cuyo objetivo, como se recoge en la propia portada de esta página web (https://aprendoencasa.educacion.es/), es «canalizar y facilitar recursos, herramientas y aplicaciones de calidad al profesorado, a las familias y al alumnado» (MEFP, 2020). El hecho de que se haya podido crear este canal tan rápidamente muestra que ya existe (existía) en nuestro país un gran bagaje de enseñanza obligatoria a distancia; quizá no tan completo como el existente en el ámbito universitario, pero definitivamente asociado a la tendencia a «virtualizar» el aprendizaje también en contextos educativos dirigidos a menores de edad.

Además de analizar la tendencia a la «virtualización» en España, es importante conocer la situación en otros países. Sería lógico pensar que la existencia de modelos educativos de enseñanza secundaria a distancia en otros países puede terminar influyendo en el nuestro. Por consiguiente, es de interés ofrecer algunos datos de otros países a modo de panorámica general. Encontramos, en este sentido, que 30 de los estados de EE. UU. ofrecen la posibilidad de estudiar en institutos completamente online, por lo que cerca de 4.500 .000 estudiantes norteamericanos han optado por dicha opción (Onlineschools, s. f.). Estas cifras contrastan con las de países como Italia, Francia, Portugal, Rusia, China o Corea del Sur, donde no se ofrece una educación secundaria a distancia; al menos no para menores de edad. México, donde la educación secundaria online surgió en 1981, supone una diferencia, puesto que se ofrece para cualquier persona de 15 años o más que no supere los estudios obligatorios a la edad estipulada. En Reino Unido, por su parte, 40.000 menores ingleses cambian las aulas físicas por sus casas (Carey, 2015), bien por asistir a institutos virtuales, bien por ser educados por sus padres. Y es precisamente esta última opción, la de estudiar en casa o homeschooling, la que escogen países como Argentina, Francia o, como veremos a continuación, EE. UU. Antes, cabe decir que, en España, pese a no tener una legislación que regule esta práctica -y a falta de un censo o registro oficial-, se calcula que hay entre 600 y 1.200 familias que ofrecen a sus hijos una educación en casa (Cabo, 2009).

\subsection{Propuestas pedagógicas de enseñanza virtual}

Teniendo en cuenta que el presente trabajo pretende establecer el diseño de una investigación que permitiría, en último lugar, proponer planes de actuación para el posible desarrollo de la enseñanza secundaria virtual -tal y como detallaremos en el apartado de objetivos-, parece pertinente explorar una alternativa a la enseñanza tradicional, el homeschooling, que consiste en «la acción pedagógica previamente planificada y ejecutada por los progenitores 
o tutores que aplican un proceso de aprendizaje» (Avalos-Obregón et al. , 2018, p. 209). Como su nombre indica, el término homeschooling se refiere al hecho de educar desde casa, al margen de la enseñanza tradicional en escuelas.

El homeschooling es el modelo de mayor crecimiento en EE. UU., donde en 2015 el número de estudiantes escolarizados de este modo ascendía a 2.200.000 (Ray, 2020). Sin embargo, esta práctica no está demasiado extendida en el continente europeo, aunque sí la reconocen Bélgica, Dinamarca, Francia, Italia, Luxemburgo, Noruega, Portugal, la mayoría de cantones suizos, Reino Unido y Austria. En cuanto a España, «la ley no reconoce la educación al margen del sistema escolar» (Valle, 2011, párr. 19).

A pesar de que nuestro país no -o todavía no- contempla esta forma de educación, teniendo en cuenta que constituye un modelo emergente en muchos otros, consideramos necesario analizar las razones que llevan a las familias a elegirlo, es decir, cuáles son sus principales ventajas y por qué está funcionando en otros contextos culturales, por si se diese el caso de que se popularizase en España hasta el punto de que la sociedad exigiese una reglamentación al respecto.

El modelo homeschooling tiene sus orígenes en el país en el que todavía hoy cuenta con el mayor número de partidarios, EE. UU. Para muchas familias, supuso una salida a la masificación y a la falta de personalización de las escuelas corrientes a finales de los setenta y principios de los ochenta (Gaither, 2008). En este punto podemos identificar la primera «clave» del éxito: la atención personalizada y las posibilidades de flexibilización. En lo que respecta a este concepto de «flexibilización», debemos destacar que, en muchos casos, el modelo homeschooling permite que las tareas se puedan realizar en un menor tiempo que en la escuela tradicional (Cobin, 2004). Si bien en las escuelas hay que contar con el tiempo de desplazamiento de los estudiantes y con una serie de restricciones organizativas -que en buena medida son también beneficiosas-, un modelo a distancia permitiría un ritmo completamente diferente.

Otra ventaja de este tipo de educación, que respalda nuestra idea de la conveniencia de desarrollar una reglamentación que contemple la educación secundaria virtual en España, es la autorresponsabilidad. Se trata de otro aspecto observable en los estudiantes de homeschooling, que aprenden a ser sus propios docentes y a ser capaces de realizar sus tareas con poca o ninguna supervisión (Cobin, 2004). El homeschooling contribuye al desarrollo responsable y autónomo del alumnado, necesario para su futuro tanto en el plano personal como en el profesional. Muchos estudios han analizado, no obstante, la falta de relaciones sociales entre iguales.

En cualquier caso, lo que sí debemos tener claro es que esta forma de educación en casa no es completamente libre, sino que existe una reglamentación que, entre otras cosas, obliga a los progenitores a dejar constancia oficial de los

\section{El bomeschooling contribuye al desarrollo responsable y autónomo del alumnado, necesario para su futuro tanto en el plano personal como en el profesional}


avances y de la evolución de sus hijos, con el objetivo de comprobar el progreso de estos (Valle, 2011). Por otra parte, y es en este aspecto donde nuestro interés por la educación secundaria virtual adquiere especial importancia, existe un alto porcentaje de alumnado de homeschooling que se ha pasado a la enseñanza reglada modalidad online o semipresencial, ya existente en sus países. De hecho, una de las principales razones que los ha llevado a ello es su facilidad y soltura en el empleo de las TIC (Cavanaugh, 2009). El hecho de ser escolarizados en casa ha impulsado su competencia digital. Por lo tanto, se evidencia que en otros países hay demanda de este tipo de enseñanza reglada virtual, lo cual nos lleva a pensar, una vez más y en vista de las circunstancias ocasionadas por la crisis de la COVID-19, que podría funcionar, ser necesaria y tener buena acogida en el nuestro.

Además del modelo homeschooling, conviene repasar otras propuestas pedagógicas que, de alguna manera, puedan llegar a contribuir a la generalización de la enseñanza secundaria virtual. En este sentido, destaca el modelo impulsado por Jonathan Bergmann -y potenciado en España por Raúl Santiago, entre otros-, denominado flipped classroom. El flipped classroom o «aula invertida» consiste en que los estudiantes reciban la explicación del docente en sus propias casas mediante vídeos que este graba previamente; de esta manera, el tiempo de la clase -bien sea presencial o virtual- se invierte en la parte práctica del aprendizaje. Este modelo es considerado por el propio Bergmann (Grupo MT Educación y Formación, 2016) como «un gran modelo para España», y, precisamente, España es, como dice Raúl Santiago, «uno de los países donde más se hace flipped classroom» (Díaz, 2018, párr. 15). Similar a esta metodología, existe también el blended learning, «aprendizaje híbrido» o «aprendizaje semipresencial», especialmente importante en el ámbito universitario (Gisbert et al., 2018), y en el que convergen los recursos y las metodologías del aprendizaje presencial y del aprendizaje a distancia al cuestionarse que pueda existir una educación $100 \%$ presencial o $100 \%$ a distancia (García Aretio, 2018).

Otras iniciativas que permiten a la población adolescente el aprendizaje a distancia vienen de la mano de docentes que comparten vídeos didácticos en YouTube, como unProfesor (s. f.); mapas mentales en Instagram, como los de @elhuertoliterario (véase Romero, 2019); o explicaciones en blogs, como www.apuntesdelengua.com (Hernández, s. f.). También surgen propuestas de gamificación (educación lúdica) 100 \% digitales, como el proyecto de escape room digital que obtuvo el premio a la Mejor Experiencia con Metodologías Activas de SIMO Educación 2019 (Educación 3.0, 2019), en el cual estudiantes de 261 centros educativos españoles y de 10 extranjeros resolvieron retos interdisciplinares. Además de todo ello, encontramos programas de ámbito autonómico como Carmenta (Castilla-La Mancha) o E-Dixgal (Galicia), de sustitución de los libros de texto por tabletas electrónicas en determinadas asignaturas y cursos.

\subsection{Papel de las familias en la educación secundaria virtual}

Una vez revisadas las opciones metodológicas y los entornos de aprendizaje que podrían facilitar la implementación de una educación secundaria virtual en España y, tras haber considerado las aportaciones de otros países en esta materia, consideramos necesario hacer 
referencia a uno de los agentes educativos más importantes: las familias. Sin su colaboración, tal y como se mencionaba en el apartado anterior, la labor educativa no llega a alcanzar su máximo potencial.

No obstante, son muchas las personas que se preguntan hasta qué punto las familias deben estar formadas para ayudar a sus hijos con las tareas -puesto que es lo que suelen realizar en casa- o con su trayectoria académica en general, olvidando que ser partícipe de la educación de sus hijos no consiste solamente en eso. Siguiendo a Orlanda Varela, «el padre se tiene que poner en la posición del entrenador. Un entrenador no corre con el jugador ni tiene que comer las mismas calorías, ni, por supuesto, sufrir sus lesiones. Un entrenador tiene dos funciones fundamentales: organizar y asesorar con el objetivo de mejorar el rendimiento» (Silió, 2013, párr. 3).

Destacamos, asimismo, que es imprescindible conseguir esta implicación parental en la etapa de educación secundaria, ya que se ha evidenciado que existe una "progresiva disminución de implicación parental en la educación con el paso de los cursos» (Valle et al., 2016, p. 491). Los adolescentes siguen necesitando apoyo familiar, más que nunca, por la fragilidad de la etapa en la que se encuentran. Este apoyo familiar es sumamente relevante, puesto que está relacionado con la satisfacción, en general, de la persona adolescente (Rodríguez-Fernández et al., 2016) y es de sobra conocido que un individuo adolescente que esté satisfecho rendirá más (Schiller y Hinton, 2015).

Sin embargo, no solamente debemos prestar atención a la formación -o falta de formación- de las familias, sino también a los lugares de residencia de estas. Según el estudio de McQuiggan et al. (2017), la tasa de alumnado homeschooler residente en zonas rurales era -en 2016- mayor que la tasa de alumnado del mismo tipo residente en ciudades o zonas suburbanas, lo que podría evidenciar que en estas zonas la enseñanza presencial es menos accesible.

Otros estudios, como el realizado por Navarro y Martínez de Anguita (2008), destacan la idoneidad de un modelo de escolarización virtual o mixta (blended-learning) para aquellos estudiantes de zonas rurales, tras haberse evidenciado grandes diferencias en aspectos como la tasa de asistencia o de abandono escolar entre este alumnado y el de las zonas urbanas. Para Mobile World Capital (2014), «la educación a distancia siempre ha sido una opción a tener en cuenta, sobre todo para aquellos que [...] viven lejos del sitio de estudio [...]. Sin embargo, internet llegó para solucionar de un plumazo todos estos problemas» (párr. 1).

Esto nos conduce a la última consideración de esta parte relacionada con los hogares y las familias: los recursos con los que estas cuentan. En este caso, al plantear una educación virtual, para garantizar que el alumnado pueda continuar su formación en línea, con «recursos" nos referiremos principalmente a un ordenador con acceso a internet. La crisis del coronavirus ha evidenciado que en España existen todavía 55.000 familias sin ordenador o 
acceso a internet para poder continuar con las clases (Vallespín, 2020a), por lo que la brecha digital es muy grande. A pesar de esto, la declaración del estado de alarma ha hecho que toda España se haya visto forzada a continuar las clases a distancia de modo improvisado. Una preparación previa de todo el país con vistas a este tipo de educación hubiese, al menos, podido reducir la improvisación que ha quedado al descubierto. De cualquier modo, las familias han dado la voz de alarma y se han tenido que adoptar medidas extraordinarias, tales como proporcionar equipos o acceso a internet a los hogares, en ocasiones a cuenta de los propios ayuntamientos (Pérez, 2020).

A la hora de diseñar de forma cautelosa la educación secundaria virtual sería esencial ser plenamente conscientes de la existencia -cada vez mayor- de la brecha digital, para poder así paliar las desigualdades en la medida de lo posible. Además, no podemos olvidar que en muchos hogares donde sí hay ordenador se cuenta solamente con uno para compartir entre todos los miembros de la unidad familiar (Álvarez, 2020). En esos casos, muchos estu-

A la hora de diseñar de forma cautelosa la educación secundaria virtual sería esencial ser plenamente conscientes de la existencia -cada vez mayor- de la brecha digital, para poder así paliar las desigualdades en la medida de lo posible diantes han optado por hacer uso de sus teléfonos móviles con acceso a internet para realizar las tareas educativas, puesto que «el rápido crecimiento de las aplicaciones móviles ha ampliado en gran medida las oportunidades de aprendizaje con dispositivos móviles» (Muñoz-Reyes, 2015, párr. 5).

De cualquier modo, incluso en el caso de que todo el país estuviese digitalmente preparado para llevar las aulas a las casas, la cuarentena, en palabras de García (2020), «evidencia las deficiencias digitales del sistema, pese a la gran cantidad de recursos disponibles y la buena voluntad de docentes y alumnos» (párr. 1). Esto refleja la pura realidad: no podemos llevar la educación de nuestros adolescentes a los hogares sin antes diseñar cuidadosamente un modelo de enseñanza secundaria virtual sólido. A su vez, esto no podrá realizarse sin antes llevar a cabo estudios de caso que permitan analizar la preparación tecnológica y formativa de las comunidades educativas.

\section{Desarrollo: diseño de investigación cualitativa}

\subsection{Objetivos}

El objetivo general de la investigación que se propone/plantea es analizar la preparación tecnológica y formativa de los miembros de la comunidad educativa (profesorado, equipo directivo, progenitores o tutores y estudiantes) del IES Impulsa para afrontar una enseñanza virtual. 
Derivado de lo anterior, los objetivos específicos serían:

1. Analizar las competencias TIC del profesorado, referente a recursos y formación tecnológica, antes y después del periodo de confinamiento por la COVID-19.

2. Conocer las metodologías y los recursos desarrollados por el profesorado durante el periodo de docencia virtual y analizar su integración en el proceso de enseñanza-aprendizaje tras dicho periodo.

3. Identificar los problemas que los docentes afrontaron durante el periodo de aprendizaje virtual.

4. Concretar las características y la evolución del alumnado en lo relativo a su formación digital, a su disciplina de "gestión del aprendizaje» y a la disponibilidad de recursos tecnológicos (dispositivos, conexión a internet, etc.) tanto antes como después del periodo de enseñanza virtual.

5. Examinar el nivel de competencia digital y el grado de participación de los progenitores o tutores en la educación de los estudiantes tanto antes como durante el periodo de docencia virtual e identificar los problemas que les surgieron como consecuencia del mismo.

6. Proponer medidas y planes de actuación para el desarrollo de la enseñanza secundaria virtual en este centro.

\subsection{Estudio de caso etnográfico}

Antes de abordar las cuestiones relativas al plan de trabajo cabe justificar la elección del IES Impulsa como caso de estudio de la etnografía que se plantea. Situado en la provincia de Alicante, se trata de un instituto público de casi 700 alumnos y 73 docentes en el que se imparte docencia en ESO, bachillerato (en la rama de Ciencias y en la de Ciencias Sociales y Humanidades) y en dos módulos de formación profesional de Comercio y Marketing (uno básico y otro de grado medio).

Si bien la idea que está detrás de este trabajo sería la de, una vez implementada la investigación, poder analizar cómo de preparada está la comunidad educativa para afrontar una enseñanza secundaria virtual, el paso posterior se centraría en llegar a constituir un documento de referencia que contribuya a la posible reglamentación de tal tipo de educación. Por ello, es preciso escoger un instituto que tenga dos características imprescindibles: que sea un centro público, puesto que los recursos económicos destinados a las TIC se estiman similares en todo el territorio nacional, y que sea un instituto que ofrezca ESO y posobligatoria (bachillerato), por si la investigación descubriese diferencias significativas en cuanto a la viabilidad de una enseñanza virtual en dichos niveles. El IES Impulsa cumple con estos dos requisitos imprescindibles para ser tomado como caso de estudio. 
Cabe decir que, teniendo en cuenta que el alcance de este trabajo es limitado -su finalidad no es ofrecer un plan educativo, sino realizar una aportación al corpus literario existente sobre este tema-, la investigación postulada debería seguir un corte metodológico cualitativo, situando a los investigadores como encargados de la obtención de una información holística que permitiera comprender la realidad. Un enfoque cuantitativo, en este contexto, no sería más apropiado, pues no interesan aquí los resultados de los estudiantes, ni las cifras de unos y otros, sino que lo que interesa son las experiencias, las emociones, las sensaciones, las motivaciones, etc. $Y$ todo ello se puede aprehender de un modo mejor mediante la palabra, los discursos vivenciales.

\subsection{Diseño de investigación}

En aras de recabar la información necesaria para dar respuesta a los objetivos específicos de la investigación diseñada se habrían de llevar a cabo distintas entrevistas semiestructuradas y grupos de discusión, tal y como se detallará y justificará a continuación y tras recoger los pertinentes consentimientos informados. Recordemos que, antes de llevar a la práctica tales técnicas de investigación, se deben diseñar sendos guiones y casilleros tipológicos. Los guiones deberán estar compuestos por preguntas que permitan a las personas entrevistadas ofrecer reflexiones lo más profundas posible, evitando que queden en la superficie de sus percepciones. Se pretende, pues, propiciar una "descripción densa» -sirviéndonos del término acuñado por Gilbert Ryle (Geertz, 1983)-.

Para cubrir los objetivos específicos número uno y dos se llevarían a cabo seis entrevistas en profundidad, técnica oportuna -siguiendo a Taylor y Bodgan (1987)- cuando la finalidad de la investigación está definida con claridad y cuando se quiere observar la realidad a través de las vivencias de las personas entrevistadas (para conocer, en este caso, cómo de preparados estaban para la enseñanza secundaria virtual antes y después del periodo de confinamiento). La selección de los docentes participantes se habrá de realizar atendiendo al casillero tipológico que sigue a estas líneas.

Las variables elegidas para el diseño de este casillero tipológico han sido: la «edad», la «etapa educativa en la que imparten docencia», los «años de experiencia en la profesión» y el «hecho de que fueran partidarios o no de la integración de las TIC en la enseñanza». Consideramos relevante incluir la edad como variable para poder observar si existen diferencias entre las dificultades encontradas por los docentes de mayor y menor edad. Por esta razón, hemos establecido una división entre los docentes de menos de 35 años, que recibieron formación en informática en la educación reglada, aquellos entre 35 y 50 años, que vivieron únicamente los inicios de los «planes dirigidos a impulsar el uso de los ordenadores en el marco escolar» (Area, 2007, párr. 5), y aquellos mayores de 50 años, que no habrían recibido esta formación en su etapa de escolarización obligatoria. En el caso de la segunda variable, hemos establecido una división entre hombres y mujeres, para observar si se evidencia brecha digital de género, aspecto que ha sido objeto de múltiples estudios, tales como el de Moreno-Guerrero et al. (2019) o el de Flores y Roig-Vila (2017). Los años 
de experiencia como docente pueden ofrecer distintas perspectivas a la hora de abordar la enseñanza de modo virtual o mediante las TIC. Por esa razón, hemos considerado que de 0 a 2 es la etapa en la que el docente se está familiarizando con el sistema, de 3 a 10 es un rango aceptable para encontrarse en un punto intermedio y más de 10 años de experiencia son suficientes para considerar a una persona "experimentada» y asentada en el cuerpo. Por último, hemos incluido el hecho de manifestar que eran -a priori-partidarios o no del uso de las TIC en las aulas, para poder observar si existen diferencias entre las opiniones de este tipo de docentes tras haber sido forzados por la pandemia a continuar su labor mediante la enseñanza virtual en el curso 2019-2020. El resultado de cruzar todas estas variables y operacionalizarlas es el casillero tipológico de docentes que se puede ver en el cuadro 1:

Cuadro 1. Casillero tipológico de docentes

\begin{tabular}{|c|c|c|c|c|c|c|c|}
\hline & & \multicolumn{3}{|c|}{ Hombre } & \multicolumn{3}{|c|}{ Mujer } \\
\hline & & $\begin{array}{c}0-2 \\
\text { años }\end{array}$ & $\begin{array}{l}3-10 \\
\text { años }\end{array}$ & $\begin{array}{l}\text { Más de } \\
10 \text { años }\end{array}$ & $\begin{array}{c}0-2 \\
\text { años }\end{array}$ & $\begin{array}{l}3-10 \\
\text { años }\end{array}$ & $\begin{array}{l}\text { Más de } \\
10 \text { años }\end{array}$ \\
\hline \multirow{3}{*}{$\begin{array}{c}\text { Partidarios } \\
\text { de usar las } \\
\text { TIC en la } \\
\text { enseñanza }\end{array}$} & $\begin{array}{c}\text { Menos } \\
\text { de } 35 \text { años }\end{array}$ & 1 & 2 & 3 & 4 & 5 & 6 \\
\hline & $\begin{array}{c}\text { Entre } \\
35 \text { y } 50 \text { años }\end{array}$ & 7 & 8 & 9 & 10 & 11 & 12 \\
\hline & $\begin{array}{l}\text { Más de } 50 \\
\text { años }\end{array}$ & 13 & 14 & 15 & 16 & 17 & 18 \\
\hline \multirow{3}{*}{$\begin{array}{c}\text { No } \\
\text { partidarios } \\
\text { de usar las } \\
\text { TIC en la } \\
\text { enseñanza }\end{array}$} & $\begin{array}{c}\text { Menos } \\
\text { de } 35 \text { años }\end{array}$ & 19 & 20 & 21 & 22 & 23 & 24 \\
\hline & $\begin{array}{c}\text { Entre } \\
35 \text { y } 50 \text { años }\end{array}$ & 25 & 26 & 27 & 28 & 29 & 30 \\
\hline & $\begin{array}{l}\text { Más de } 50 \\
\text { años }\end{array}$ & 31 & 32 & 33 & 34 & 35 & 36 \\
\hline
\end{tabular}

Fuente: elaboración propia.

Una vez analizadas las vivencias de los docentes para conocer qué nivel de competencias TIC desarrollaron durante el periodo de confinamiento (objetivo específico número 1), y las metodologías que mantienen en su posterior actividad profesional (objetivo específico número 2), interesaría construir el discurso del profesorado para conocer los problemas que surgieron durante el periodo de aprendizaje virtual y las medidas que se adoptaron para afrontarlos (objetivo número 3). Para cubrir tal objetivo, se propone la implementación de la técnica del grupo de discusión. 
Definidos por Massot et al. (2019) como «una discusión cuidadosamente diseñada para obtener las percepciones sobre una particular área de interés [...] que capacita al investigador para alinearse con los participantes y descubrir cómo ven la realidad» (p. 335), la intención de los grupos de discusión es, según estos mismos autores, «promover la autoapertura y generar un discurso grupal para identificar distintas tendencias» (p. 335). Se ha escogido esta técnica de recogida de datos sobre otras puesto que consideramos interesante conocer no las experiencias individuales de un agente, sino el discurso generado por los grupos de docentes. El diseño resultante se recoge en la figura 1.

Figura 1. Grupos de discusión del profesorado

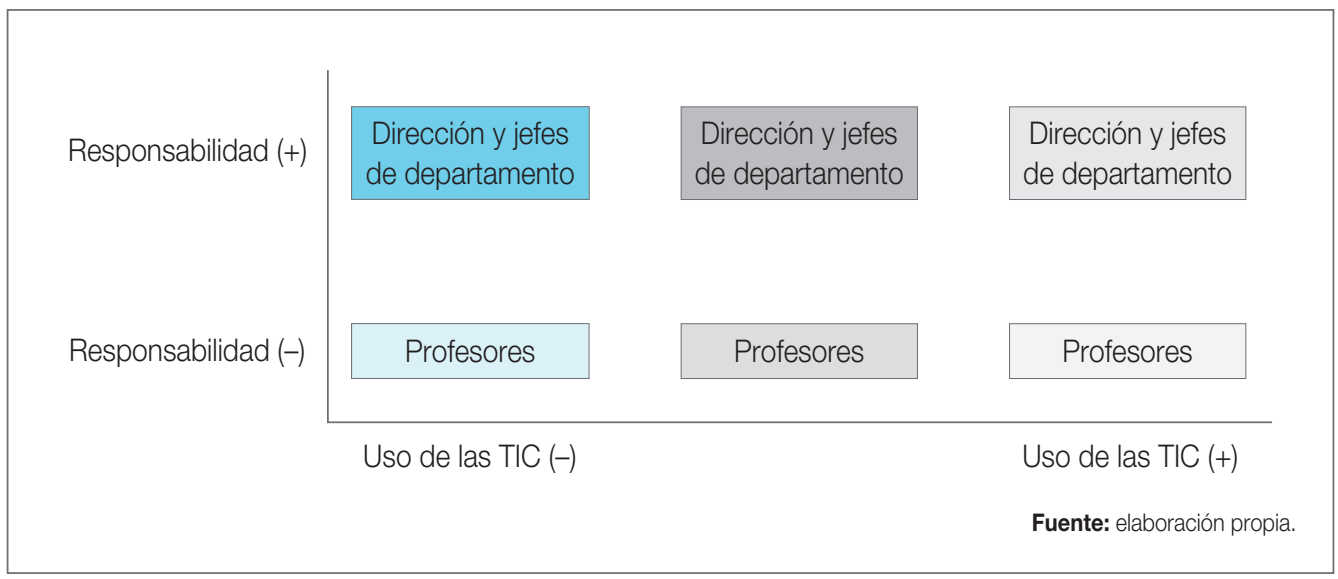

Para establecer las variables susceptibles de ser tomadas en consideración en el diseño de grupos de discusión que habrían de realizarse hemos identificado como primer eje el «nivel de responsabilidad». Hemos situado, por tanto, en lo más alto del eje al profesorado con cargo directivo en el centro (personas que ocupan los puestos de director, vicedirector, jefe de estudios y secretario) y a aquellos con cargo de jefatura de departamento, que han tenido que coordinar las acciones llevadas a cabo por los demás docentes de la misma materia en el centro y modificar las programaciones didácticas. En la posición más baja se encontrarían aquellos docentes sin cargo específico. Con respecto al segundo eje, hemos escogido la variable «uso de las TIC», situando en la parte más a la derecha a aquel profesorado que ya utilizaba las TIC con frecuencia (una o varias veces a la semana) en su desempeño laboral. En una posición intermedia hemos localizado a aquellos docentes que no empleaban las TIC tan a menudo en sus puestos de trabajo (una o dos veces al mes), pero que sí hacían uso de entornos de aprendizaje en línea, tales como el aula virtual del centro. En último lugar, se encontrarían aquellos docentes que no empleaban las TIC en su materia antes del periodo de confinamiento. A la hora de establecer la variable de fragmentación intragrupal para garantizar la heterogeneidad inclusiva, hemos decidido separar a los docentes según su «especialidad» (Ciencias o Humanidades y Ciencias Sociales). Por tanto, se 
propone la realización de los siguientes tres grupos de discusión, en el orden que se indica a continuación, para tratar de obtener un discurso lo más variado y rico posible. En el caso de que no se alcanzase la saturación de discursos, se llevarían a cabo tantos grupos de discusión como fueran necesarios de entre los que se detallan en el diseño anterior.

- G1. Siete docentes con alto uso de las TIC: dos cargos directivos (uno de la rama de Ciencias y otro de la de Humanidades y Ciencias Sociales) y cinco jefes de departamento (tres de Ciencias y dos de Humanidades y Ciencias Sociales).

- G2. Siete docentes con uso medio de las TIC (cuatro de la rama de Humanidades y Ciencias Sociales y tres de la de Ciencias).

- G3. Seis docentes con bajo uso de las TIC: un cargo directivo de la rama de Ciencias y cinco jefes de departamento (tres de Humanidades y Ciencias Sociales y dos de Ciencias).

Para dar respuesta al cuarto objetivo específico (relativo al nivel de preparación del alumnado para afrontar la educación virtual), es necesario conocer el discurso generado por el estudiantado. Por ello, se postula como conveniente la realización, en el marco de la investigación diseñada y propuesta en este artículo, de una serie de grupos de discusión, tal y como se describe en el diseño de la figura 2.

Figura 2. Grupo de discusión del alumnado



A la hora de establecer las variables para estos grupos de discusión hemos considerado como primer eje el "curso" en el que se encontraba el alumnado durante la crisis de la COVID-19, puesto que buscamos un discurso con distintas posiciones. Por esta razón, hemos situado en lo más alto de este eje al alumnado de $2 .^{\circ}$ de bachillerato, enormemente afectado por la situación debido a la prueba de acceso a la universidad que debía 
realizar tras terminar el curso. En un punto intermedio hemos localizado al alumnado de 4 . $^{\circ}$ de ESO, que estaba a punto de terminar la etapa de secundaria y obtener un título que le permitiese tomar decisiones sobre su futuro: continuar estudiando bachillerato o acceder a la formación profesional de grado medio. Finalmente, hemos situado en lo más bajo del eje al alumnado de $1 .^{\circ}$ de ESO, los más jóvenes del centro y recién llegados a la etapa de educación secundaria, a los que todavía les queda un largo camino por recorrer hasta terminarla. En lo que respecta al segundo eje, nos hemos decantado por hacer referencia a los «recursos» del alumnado, colocando más a la izquierda a aquellos con menos recursos; en este caso, se trataría de estudiantes sin ordenador ni internet. En un grupo intermedio estarían aquellos estudiantes que tuviesen, o bien internet, o bien ordenador en casa, pero no ambos. Por último, en la parte más a la derecha, hemos situado al alumnado con más recursos, es decir, que cuentan tanto con ordenador como con internet en sus hogares. De este modo tendremos una idea más clara de cómo han afrontado el hecho de que el proceso de enseñanza-aprendizaje pasase a ser virtual.

No obstante, es necesario también establecer una serie de variables de fragmentación intragrupal que permitan garantizar una heterogeneidad inclusiva dentro de cada uno de los grupos. De este modo, hemos dividido al alumnado, en primer lugar, atendiendo a su «Competencia digital», distinguiendo entre los estudiantes con una mayor competencia digital de acuerdo con los consejos orientadores del curso anterior -sobresaliente o notable-, los estudiantes con la calificación de «bien» en dicha competencia y, por último, aquellos con la calificación de «suficiente» o «insuficiente». Como segunda y última variable de fragmentación intragrupal, hemos tenido en cuenta los «itinerarios formativos» -en el caso de $4 .^{\circ}$ de ESO y $2 .^{\circ}$ de bachillerato, puesto que en $1 .^{\circ}$ de ESO no existen-.

Por tanto, se propone la realización de los siguientes grupos de discusión. Se realizarían en el orden en que están numerados para tratar de obtener un discurso tan variado y completo como sea posible. Si no se alcanzara el punto de saturación, se deberían llevar a cabo más grupos de discusión.

- G1. Seis estudiantes de $1 .^{\circ}$ de ESO sin ordenador ni internet: dos con competencia digital «buena», dos con competencia digital «sobresaliente/notable» y dos con competencia digital «suficiente/insuficiente».

- G2. Seis estudiantes de $4 .^{\circ}$ de ESO sin ordenador o internet: tres del itinerario de Enseñanzas Académicas y tres de Enseñanzas Aplicadas. De esos tres, en ambos casos habrá un estudiante con competencia digital «sobresaliente/notable», uno con competencia digital «buena» y otro con competencia digital «suficiente/insuficiente».

- G3. Seis estudiantes de $2 .^{\circ}$ de bachillerato con ordenador e internet: tres de cada itinerario (Ciencias o Humanidades y Ciencias Sociales). En ambos casos habrá un estudiante de cada «nivel» de competencia digital, como se indicaba en el grupo de discusión G2. 
- G4. Seis estudiantes de $4 .{ }^{\circ}$ de ESO sin ordenador ni internet: tres del itinerario de Enseñanzas Académicas y tres del de Enseñanzas Aplicadas. En ambos casos habrá un estudiante de cada «nivel» de competencia digital.

- G5. Seis estudiantes de $1 .^{\circ}$ de ESO con ordenador e internet: dos con competencia digital «buena», dos con competencia digital «sobresaliente/notable» y dos con competencia digital «suficiente/insuficiente».

- G6. Seis estudiantes de $2 .^{\circ}$ de bachillerato sin ordenador o internet: tres de cada itinerario (Ciencias o Humanidades y Ciencias Sociales). En ambos casos habrá un estudiante de cada «nivel» de competencia digital.

Además de conocer las competencias tecnológicas, los problemas derivados de la docencia virtual y, en general, el discurso del profesorado y del alumnado, quedarían por conocer las experiencias y puntos de vista de otro sector fundamental de la comunidad educativa: las familias. A ello se refiere el quinto objetivo específico de la investigación propuesta en este artículo. Para cubrirlo, se realizarían entrevistas en profundidad a seis padres, madres, tutores o tutoras de estudiantes, escogiendo a los entrevistados en función de un casillero tipológico elaborado a tal efecto.

En este caso, se han establecido las siguientes variables para diferenciar tipos de progenitores o tutores: "situación laboral durante la mayor parte del confinamiento», "curso en el que estudian sus hijos», «internet en casa» y «ordenador compartido». La «situación laboral» de los posibles entrevistados es una variable relevante, ya que muchos de ellos habrán sido teletrabajadores, a la vez que sus hijos estudiaban en casa, otros habrán continuado con su actividad presencial por el sector al que pertenecen y otros habrán permanecido o continuado en su hogar en situación de desempleo, expediente de regulación temporal de empleo (ERTE) o similares. El hecho de incluir la variable «curso», por su parte, es necesario por si existiese alguna diferencia sustancial en cuanto a la facilidad del alumnado a la hora de desenvolverse en un entorno completamente digital según su edad y etapa educativa. Por esta razón, hemos hecho una distinción entre $1 .^{\circ}$ de ESO $4 .^{\circ}$ de ESO y $2 .^{\circ}$ de bachillerato. Esto nos aproximaría a conocer si es viable la educación virtual para todos los cursos de educación secundaria o si sería más conveniente aplicarla a partir de una edad (como ya se hace con la ESO a distancia para mayores de edad).

Finalmente, poseer -o no- ordenador se ha considerado de importancia, puesto que esta crisis ha evidenciado más que nunca la brecha digital existente en España. Además, tal y como se mencionaba en el apartado 2.3., son muchas las familias que se han visto en la problemática de tener que «compartir un mismo dispositivo» entre varios miembros. Del mismo modo, hemos tenido en cuenta si las familias tenían o no «internet en casa» durante el periodo de docencia virtual. El resultado de cruzar todas estas variables y operacionalizarlas es el siguiente casillero tipológico (véase cuadro 2): 
Cuadro 2. Casillero tipológico de progenitores o tutores

\begin{tabular}{|c|c|c|c|c|c|}
\hline & & \multicolumn{2}{|c|}{ Internet } & \multicolumn{2}{|c|}{ No internet } \\
\hline & & $\begin{array}{l}\text { Dispositivo } \\
\text { compartido }\end{array}$ & $\begin{array}{c}\text { Dispositivo } \\
\text { propio }\end{array}$ & $\begin{array}{l}\text { Dispositivo } \\
\text { compartido }\end{array}$ & $\begin{array}{c}\text { Dispositivo } \\
\text { propio }\end{array}$ \\
\hline \multirow{3}{*}{ Teletrabajo } & $\begin{array}{c}2 .^{\circ} \text { de } \\
\text { bachillerato }\end{array}$ & 1 & 2 & 3 & 4 \\
\hline & $4 .^{\circ}$ de ESO & 5 & 6 & 7 & 8 \\
\hline & $1 .^{\circ}$ de ESO & 9 & 10 & 11 & 12 \\
\hline \multirow{3}{*}{$\begin{array}{l}\text { Actividad } \\
\text { laboral } \\
\text { presencial }\end{array}$} & $\begin{array}{c}2 .^{\circ} \text { de } \\
\text { bachillerato }\end{array}$ & 13 & 14 & 15 & 16 \\
\hline & $4 .^{\circ}$ de ESO & 17 & 18 & 19 & 20 \\
\hline & $1 .^{\circ}$ de ESO & 21 & 22 & 23 & 24 \\
\hline \multirow{3}{*}{$\begin{array}{c}\text { No } \\
\text { actividad } \\
\text { laboral }\end{array}$} & $\begin{array}{c}2 .^{\circ} \text { de } \\
\text { bachillerato }\end{array}$ & 25 & 26 & 27 & 28 \\
\hline & $4 .^{\circ}$ de ESO & 29 & 30 & 31 & 32 \\
\hline & $1 .^{\circ}$ de ESO & 33 & 34 & 35 & 36 \\
\hline
\end{tabular}

Fuente: elaboración propia.

A la hora de dar respuesta al último objetivo específico planteado en este diseño de investigación, se llevaría a cabo un grupo triangular, una técnica a medio camino entre las entrevistas y los grupos de discusión, con discursos en los que predominan las funciones fática y referencial (Ruiz, 2012). En nuestro caso, proponemos un grupo triangular en el que se confrontasen los discursos generados por los miembros del equipo directivo y por los miembros de los referidos grupos de discusión del profesorado.

Tomando como referencia el grupo de discusión previo del profesorado, para llevar a cabo este grupo triangular, escogeríamos a distintos miembros de la comunidad educativa para poder buscar propuestas de mejora y ofrecer soluciones.

Por tanto, se realizaría un grupo triangular formado por un miembro del equipo directivo, un docente en una posición alta del eje de uso de las TIC y un padre o una madre de los entrevistados siguiendo el casillero diseñado a tal efecto (que representaría, a su vez, de forma vicaria, al estudiante). 


\subsection{Plan de explotación de los resultados}

Siguiendo el procedimiento habitual en los estudios cualitativos, el análisis de los datos obtenidos del desarrollo del diseño de investigación propuesto no se realizaría al finalizar la etnografía, sino que su revisión e interpretación, a lo largo del proceso de investigación llevado a cabo, iría arrojando la luz necesaria para determinar si es necesario modificar o ampliar el diseño de la misma e ir construyendo la narración etnográfica. Para ello se deberían transcribir las entrevistas y los grupos de discusión, llevándose a cabo después la codificación y sistematización de los datos que se habrán obtenido. Durante todo este proceso se irían tomando notas y escribiendo fragmentos de lo que constituirá, llegado el momento, el informe final. En este se dará cuenta de las conclusiones alcanzadas y de la manera en que se han obtenido y analizado los datos. Este informe, además, deberá permitir al lector tener una comprensión del caso de estudio suficiente como para conformar su propia reflexión al respecto (Massot et al., 2019). Finalmente, una vez se hayan descrito en profundidad las distintas dimensiones del caso y se haya construido la narración, interesará sopesar la posibilidad de generalizar o exportar a otros casos los resultados de la etnografía a la que la aplicación de esta propuesta de investigación dará lugar. Cabe decir que la información que se obtenga a través de las técnicas de investigación cuya implementación se plantea y, sobre todo, el análisis derivado de dicha información permitirán, previsiblemente, cubrir los objetivos planteados en el apartado 3.1.

\section{Conclusiones}

Una vez llegados a este punto de nuestro trabajo, en el que hemos hecho una propuesta de investigación de carácter cualitativo para analizar el funcionamiento de la enseñanza secundaria a distancia durante la pandemia de la COVID-19 en un instituto público, el IES Impulsa, nos gustaría establecer unas conclusiones relacionadas con el diseño de la investigación y los agentes implicados.

Si bien es cierto que las situaciones excepcionales, tales como la que hemos vivido, requieren medidas excepcionales, no debemos olvidar que es necesario dejar el menor lugar posible a la improvisación, especialmente cuando se trata de uno de los pilares fundamentales de la sociedad: la educación. Por este motivo, el último objetivo de la investigación diseñada adquiere especial importancia: es necesario proponer planes de actuación que nos permitan estar preparados para afrontar posibles escenarios futuros. La educación virtual en España no es un terreno desconocido en el ámbito universitario; sin embargo, existe todavía un largo camino por recorrer en lo que respecta a otros niveles educativos.

Por consiguiente, pretendemos que la excepcionalidad que nos ha tocado vivir sirva como experiencia para fundamentar el diseño metodológico de planes educativos sólidos que atiendan a las vivencias de los miembros de la comunidad educativa. Por esta razón, en el estudio planteado se tiene en cuenta, en primer lugar, a las familias, que se vieron obligadas a enfrentarse a una situación nueva en lo respectivo a la educación de sus hijos, muchas veces a 
la espera de instrucciones y/o recursos que les garantizasen poder continuar escolarizados. También hemos considerado a los docentes, que han demostrado ser agentes esenciales sin los cuales no sería posible concebir la educación. Estos son, no obstante, a menudo olvidados a la hora de diseñar planes educativos. Durante la pandemia vivida, la frustración ha inundado los claustros debido a la falta de recursos, de un reglamento sólido, de instrucciones de carácter más práctico e inmediato y, en definitiva, de preparación previa. Por último, se ha tenido en cuenta a los estudiantes, verdaderos protagonistas del proceso de enseñanza-aprendizaje y principales afectados por los cambios del último trimestre y por la falta de preparación de un modelo de educación secundaria a distancia. Cada uno de los estudiantes de un centro educativo tiene unas características y necesidades diferentes, bien sea en aulas presenciales o no presenciales, y todos ellos deberían ser una prioridad si el objetivo es conseguir un sistema educativo flexible, preparado y que garantice la igualdad de oportunidades.

Indistintamente de cuáles sean los discursos que se recaben, la aplicación del diseño de investigación propuesto permitiría cubrir los objetivos planteados y, por consiguiente, contribuir al desarrollo de la literatura necesaria para dar respuesta al problema nuclear que planteábamos al inicio de este documento: la sociedad y las circunstancias requieren de una reglamentación de la educación secundaria virtual que atienda a la realidad de centros educativos como el que serviría de caso de estudio en la etnografía aquí propuesta.

\section{Referencias bibliográficas}

Adell, J. y Sales A. (1999). El profesor online: elementos para la definición de un nuevo rol docente. Comunicación presentada en el Curso de Formación de Formadores Virtuales, Valencia, España. Curso organizado por la Cámara de Comercio de Valencia.

AEPD. (2018). Informe sobre la utilización por parte de profesores y alumnos de aplicaciones que almacenan datos en la nube con sistemas ajenos a las plataformas educativas. https://www.aepd.es/es/prensa-y-comuni cacion/notas-de-prensa/la-aepd-publicaun-informe-sobre-la-utilizacion-de

Álvarez Bardón, A. (11 de abril de 2020). Alumnos sin acceso a la educación a distancia: la pandemia saca a la luz grandes desigualdades. Diario Sur. https://www.diariosur.es/

Area, M. (13 de mayo de 2007). Historia de la informática educativa en España (I). Los años ochenta o la edad de la inocencia. http://orde nadoresenelaula.blogspot.com
Avalos-Obregón, M. L., Avalos-Obregón, M. ${ }^{a}$ D. y Cazar del Pozo, F. (2018). Homeschooling una alternativa en educación. Polo del Conocimiento, 3(10), 206-222. https://doi.org/10. 23857/pc.v3i10.743

Cabo González, C. (2009). El homeschooling en España: descripción y análisis del fenómeno (Tesis doctoral). Universidad de Oviedo, Asturias, España.

Carey, T. (1 de octubre de 2015). Would you let your child be taught at home by a computer? More pupils are having school lessons over the internet-but will it turn them into lonely misfits? Daily Mail. https://www. dailymail.co.uk/

Cavanaugh, C. (2009). Effectiveness of cyber charters: a review of research on learnings. Tech Trends, 53(4), 28-31.

CIERD. (s. f.). Nuestro centro. https://www.edu cacionyfp.gob.es/

Cobin, J. (27 de octubre de 2004). Homeschooling is a doable alternative. http://www.poli cyofliberty.net/papers.php 
Consejería de Educación, Cultura y Deportes de Castilla-La Mancha. (2020). Instrucciones de 13 de abril de 2020 de la Consejería de Educación, Cultura y Deportes sobre medidas educativas para el desarrollo del tercer trimestre del curso 2019-2020, ante la situación de estado de alarma provocada por causa del brote del virus COVID-19. Toledo: Junta de Comunidades de Castilla-La Mancha. https:// nube.castillalamancha.es

Díaz Sotero, P. (26 de junio de 2018). Raúl Santiago: «España es uno de los países donde más se hace flipped classroom». http://actua lidaddocente.cece.es

Educación 3.0. (11 de octubre de 2019). ¡Ganadores de los VII Premios a la Innovación Educativa SIMO EDUCACIÓN 2019! https:// www.educaciontrespuntocero.com

Flores Lueg, C. y Roig-Vila, R. (2017). El género y su incidencia en el nivel de competencia digital autopercibido por estudiantes de Pedagogía. International Journal of Educational Research and Innovation, 8, 79-86.

Gaither, M. (2008). Homeschool: An American History. Palgrave Macmillan.

García, J. G. (22 de marzo de 2020). Colegios. La odisea de la educación online impuesta por el coronavirus. Retina El País. https://re tina.elpais.com/

García Aretio, L. (2018). Blended learning y la convergencia entre la educación presencial y a distancia. RIED. Revista lberoamericana de Educación a Distancia, 21(11), 195-213. https://doi.org/10.5944/ried.21.1.19683

Geertz, C. (1983). La interpretación de las culturas (A. L. Bixio, trad.). Gedisa. (Original publicado en 1973).

Gisbert Cervera, M., Benito Crosetti, B. de, Pérez Garcies, A. y Salinas Ibáñez, J. (2018). Blended learning, más allá de la clase presencial. RIED. Revista Iberoamericana de Educación a Distancia, 21(1), 195-213.

Grupo MT Educación y Formación. (31 de octubre de 2016). Intervención de Jon Bergmann en
Cuatro explicando el enfoque flipped [vídeo]. YouTube. https://youtu.be/gfOFhdTXhUE

Hernández Ortega, J. (s. f.). Apuntes de Lengua [blog]. http://www.apuntesdelengua.com

INE. (2018). Anuario Estadístico de España 2018. http://www.ine.es/prodyser/pubweb/anua rios_mnu.htm

Ley orgánica 14/1970, de 4 de agosto, general de educación y financiamiento de la reforma educativa (BOE núm. 187, de 6 de agosto de 1970, p. 12.532).

Ley orgánica 2/2006, de 3 de mayo, de educación (BOE núm. 106, de 4 de mayo de 2006, p. 16).

Massot Lafon, I., Dorio Alcaraz, I. y Sabariego Puig, M. (2019). Estrategias de recogida y análisis de la información. En R. Bisquerra Alzina, Metodología de la investigación educativa (pp. 321-358). La Muralla.

McQuiggan, M., Megra, M. y Grady, S. (2017). Parent and Family Involvement in Education: Results from the National Household Education Surveys Program of 2016. National Center for Education Statistics.

MEFP. (2018a). Informe 2018 sobre el estado del sistema educativo. Curso 2016-2017. http:// www.educacionyfp.gob.es/educacion/mc/ cee/publicaciones/informes-del-sistemaeducativo/informe-2018.html

MEFP. (2018b). Memoria de actividades CIDEAD curso 2017/18. https://www.educacionyfp. gob.es/dam/jcr:4beca82a-5c64-4753-b44 3-34eb39241af9/MEMORIA\%20CIDEAD\%2 OCURSO\%202017-18.pdf

MEFP. (2019). Datos y cifras. Curso escolar 20192020. Secretaría General Técnica.

MEFP.(2020).AprendoEnCasa. https://aprendo encasa.educacion.es/

Ministerio de Universidades. (2020). Datos y cifras del sistema universitario español. http:// www.educacionyfp.gob.es/dam/jcr:b9e 82c7a-1174-45ab-8191-c8b7e626f5aa/ informe-datos-y-cifras-del-sistema-universita rio-espa-ol-2019-2020-corregido.pdf 
Mobile World Capital. (21 de octubre de 2014). Educación online vs. educación tradicional, ¿es igual de efectiva? https://mobileworldca pital.com

Moreno-Guerrero, A., Fernández Mora, M. ${ }^{a}$ A. y Alonso García, S. (2019). Influencia del género en la competencia digital docente. Revista Espacios, 40(41), 30-45.

Muñoz-Reyes, C. (18 de febrero de 2015). Disminuyendo la brecha digital en las comunidades rurales a través del mobile learning. https://observatorio.profuturo.education

Nadeu, F. (14 de abril de 2020). Educación comienza a repartir ordenadores entre alumnos que no tengan. el Periódico. https://www. elperiodico.com/

Navarro, M. y Martínez de Anguita, P. (2008). Evaluación de un modelo blend-learning de educación para el desarrollo rural adaptado a la realidad colombiana. Revista Iberoamericana de Educación, 45(4), 1-11.

Onlineschools.org. (s. f.). Guide to Online High School. https://www.onlineschools.org/

Orden EFP/365/2020, de 22 de abril, por la que se establecen el marco y las directrices de actuación para el tercer trimestre del curso 2019-2020 y el inicio del curso 2020-2021, ante la situación de crisis ocasionada por el COVID-19 (BOE núm. 114, de 24 de abril de 2020, p. 7). Ministerio de Educación y Formación Profesional.

Pérez, A. (9 de abril de 2020). Coronavirus en Coruña. Receta contra la brecha digital. La Opinión Coruña. https://www.laopinioncoruna.es

Ray, B. D. (2020). Research Facts on Homeschooling. https://www.nheri.org

Rodríguez-Fernández, A., Ramos-Díaz, E., Ros, I., Fernández-Zabala, A. y Revuelta, L. (2016). Bienestar subjetivo en la adolescencia: el papel de la resiliencia, el autoconcepto y el apoyo social percibido. Suma Psicológica, 23(1), 60-69.

Romero, A. (2019). El huerto literario. Materiales educativos. https://www.elhuertoliterario.com

Ruiz Ruiz, J. (2012). El grupo triangular: reflexiones metodológicas en torno a dos expe- riencias de investigación. EMPIRIA. Revista de Metodología de Ciencias Sociales, 24, 141-162. https://doi.org/10.5944/empiria. 24.2012 .846

Schiller, L. y Hinton, C. (30 de julio de 2015). It's true: happier students get higher grades. The Conversation. https://theconversation.com

Silió, E. (17 de enero de 2013). Ayudarle a hacer los deberes no es ayudarle. El País. https:// elpais.com

Taylor, S. J. y Bogdan, R. (1987). Introducción a los métodos cualitativos de investigación: la búsqueda de significados (J. Piatigorsky, trad.). Paidós. (Original publicado en 1984).

U-Multirank (s. f.). University Comparison. https:// www.umultirank.org

unProfesor (s. f.). Consejos para estudiar. https://www.unprofesor.com/consejospara-estudiar/

Valle, A., Regueiro, B., Núñez, J. C., Suárez, N., Freire, C. y Ferradás, M. (2016). Percepción de la implicación parental en los deberes escolares y rendimiento académico en estudiantes de secundaria. Revista Española de Pedagogía, 265, 481-498.

Valle Aparicio, J. E. (2011). Enseñar en casa o en la escuela. La doctrina legal sobre el homeschooling en España. Perfiles Educativos, 34(138), 167-182.

Valles, M. S. (2000). La grounded theory y el análisis cualitativo por ordenador. En M. García Ferrando, J. Ibáñez y F. Alvira (Comps.), El análisis de la realidad social: métodos y técnicas de investigación (pp. 575-604). Alianza.

Vallespín, I. (8 de abril de 2020a). Educación detecta 55.000 familias sin ordenador o sin conexión para poder seguir las clases a distancia. El País. https://elpais.com

Vallespín, I. (14 de abril de 2020b). Educación solo asume la mitad de los ordenadores de los alumnos vulnerables. El País. https:// elpais.com/

Xunta de Galicia. (15 de abril de 2020). Galicia lamenta a indefinición do Ministerio de Educación para establecer as condición de promoción e titulación. https://www.edu.xunta.gal 